\title{
Association of body mass index with risk of acute myocardial infarction and mortality in Norwegian male and female patients with suspected stable angina pectoris: a prospective cohort study
}

Heidi Borgeraas ${ }^{1,2,3^{*}}$, Jens Kristoffer Hertel ${ }^{1}$, Gard Frodahl Tveitevåg Svingen ${ }^{3}$, Reinhard Seifert ${ }^{4}$, Eva Kristine Ringdal Pedersen ${ }^{3}$, Hall Schartum-Hansen ${ }^{4}$, Jøran Hjelmesæth ${ }^{1,2+}$ and Ottar Nygård ${ }^{3,4+}$

\begin{abstract}
Background: A number of previous studies have suggested that overweight or obese patients with coronary artery disease (CAD) may have lower morbidity and mortality than their leaner counterparts. Few studies have addressed possible gender differences, and the results are conflicting. We examined the association between body mass index (BMI) and risk of acute myocardial infarction (AMI), cardiovascular (CV) death and all-cause mortality in men and women with suspected stable angina pectoris.

Method: The cohort included 4164 patients with suspected stable angina undergoing elective coronary angiography between 2000 and 2004. Events were registered until the end of 2006. Hazard ratios (HR) (95\% confidence intervals) were estimated using Cox regression by comparing normal weight $\left(18.5-24.9 \mathrm{~kg} / \mathrm{m}^{2}\right)$ with overweight $\left(25-29.9 \mathrm{~kg} / \mathrm{m}^{2}\right)$ and obese $\left(\geq 30 \mathrm{~kg} / \mathrm{m}^{2}\right)$ patients. Underweight $\left(<18.5 \mathrm{~kg} / \mathrm{m}^{2}\right)$ patients were excluded from the study.

Results: Of 4131 patients with complete data, $72 \%$ were males and $75 \%$ were diagnosed with significant CAD. The mean (standard deviation (SD)) age in the total population was 62 (10) years. Mean (SD) BMl was $26.8(3.9) \mathrm{kg} / \mathrm{m}^{2}, 34 \%$ was normal weight, $48 \%$ overweight and 19\% obese. During follow up, a total of 337 (8.2\%) experienced an AMI and 302 (7.3\%) patients died, of whom 165 (4.0\%) died from cardiovascular causes. We observed a significant interaction between BMI groups and gender with regards to risk of AMI $(p=0.011)$ and CV death $(p=0.031)$, but not to risk of all-cause mortality; obese men had a multivariate adjusted increased risk of $\operatorname{AMI}(\operatorname{HR} 1.80(1.28,2.52))$ and CV death (HR $1.60(1.00,2.55))$ compared to normal weight men. By contrast, overweight women had a decreased risk of $\mathrm{AMI}(\mathrm{HR} 0.56(0.33,0.98))$ compared to normal weight women. The risk of all-cause mortality did not differ between BMI categories.
\end{abstract}

Conclusion: Compared with normal weight subjects, obese men had an increased risk of AMI and CV death, while overweight women had a decreased risk of AMI. These findings may potentially explain some of the result variation in previous studies reporting on the obesity paradox.

Trial registration: Clinicaltrials.gov Identifier: NCT00354081

Keywords: Acute myocardial infarction, Body mass index, Cardiovascular disease, Obesity paradox

\footnotetext{
* Correspondence: heibor@siv.no

${ }^{\dagger}$ Equal contributors

'Morbid Obesity Center, Vestfold Hospital Trust, Tønsberg, Norway

${ }^{2}$ Institute of Clinical Medicine, University of Oslo, Oslo, Norway

Full list of author information is available at the end of the article
} 


\section{Background}

Cardiovascular disease (CVD) is the leading cause of death globally: the majority dying from ischemic heart disease [1]. Overweight and obesity, most commonly defined according to body mass index (BMI), has been characterized as a major modifiable risk factor for cardiovascular (CV) morbidity and mortality by the American Heart Association and the American College of Cardiology [2].

As recently reviewed, some studies of patients with coronary artery disease (CAD) suggest that being overweight or obese has beneficial effects in terms of reduced risk of CV events and/or mortality; a phenomenon known as the obesity paradox. However, there is no broad consensus regarding the obesity paradox, as several studies are unsupportive of this conclusion [3].

Moreover, despite the fact that both body fat percentage and distribution vary by gender [4], only a limited number of studies among patients with CAD or suspected CAD have examined the association between BMI and risk of coronary events and mortality in men and women separately, and the reported results are conflicting [5-9]. There is, however, a tendency towards a non-disadvantageous [5,6,9] or even a beneficial [7] effect of overweight and obesity among women, while obesity appears to increase the risk of coronary events in men $[5,6]$.

In the present study we examined the association between BMI and risk of incident acute myocardial infarction (AMI), CV death and all-cause mortality in a large population of men and women with suspected CAD. We hypothesized that overweight and/or obesity, as compared to normal weight, was associated with an increased risk of AMI, CV death and all-cause mortality among men, but not among women.

\section{Methods}

\section{Study design and patient population}

The patients recruited for the present investigation are described in detail elsewhere [10]. In brief, 4164 patients undergoing elective coronary angiography for suspected stable angina pectoris were recruited from two university hospitals in Western Norway from January 2000 to April 2004. Of these patients, 2573 (62.0\%) were enrolled in the Western Norway B Vitamin Intervention Trial (WENBIT) which studied the prognostic impact of B-vitamin supplementation upon incident $\mathrm{CV}$ events and mortality (clinicaltrials.gov Identifier: NCT00354081) [11]. Patients for whom there was no BMI data $(n=3)$ were excluded from the study, as were underweight patients $\left(B M I<18.5 \mathrm{~kg} / \mathrm{m}^{2}\right)$ $(n=30)$.This left a total of 4131 subjects eligible for the analyses.

The study protocol met the mandate of the Helsinki Declaration, and was approved by the Western Norway Regional Committee for Medical and Health Research
Ethics and the Norwegian Data Inspectorate. Written informed consent was obtained from all participants.

\section{Baseline data and biochemical analyses}

Height, weight and blood pressure were measured at baseline by trained study personnel. BMI was calculated by dividing weight by height squared $\left(\mathrm{kg} / \mathrm{m}^{2}\right)$. Each patient provided information about medical history, risk factors and medications through a self-administered questionnaire, and all information was subsequently validated against medical records. Diabetes mellitus included type 1 and 2. Current smokers included those with self-reported current smoking, those who had quit smoking within $<1$ month and those with plasma cotinine $>85 \mathrm{ng} / \mathrm{mL}$ [12]. Patients, who reported to have quit smoking > 1 month prior to inclusion and had plasma cotinine levels $\leq 85 \mathrm{ng} / \mathrm{mL}$, were categorized as ex-smokers. Pulmonary disease included chronic obstructive lung disease, other chronic lung diseases and pulmonal hypertension. Cancer included active cancer with or without metastases. Family history of early coronary heart disease (CHD) encompassed those reporting to have at least one $1^{\text {st }}$ degree relative suffering from CHD before the age of 55 for men and 65 for women. Left ventricular ejection fraction (LVEF) was determined by ventriculography or echocardiography. The extent of CAD at angiography was scored as $0-3$ as has previously been described [11]. Baseline coronary revascularisation procedures, after baseline angiography, included percutaneous coronary intervention (PCI) and coronary artery bypass graft surgery (CABG).

Blood samples were collected by study personnel prior to angiography and stored at $-80^{\circ} \mathrm{C}$ until analysis. Serum apolipoprotein A-1 (ApoA1), apolipoprotein B (ApoB) and lipoprotein (a) $(\mathrm{Lp}(\mathrm{a}))$ were analysed on the Hitachi 917 system (Roche Diagnostics, GmbH, Mannheim, Germany). Serum C-reactive protein (CRP) was measured using a latex, high sensitive assay (Behring Diagnostics, Marburg, Germany). Plasma cotinine was measured by liquid chromatography/tandem mass spectrometry [13]. Low density lipoprotein (LDL) cholesterol was calculated using the Friedewald formula [14] and estimated glomerular filtration rate (eGFR) was estimated using the Chronic Kidney Disease Epidemiology Collaboration formula [15].

\section{Follow-up and end points}

The study participants were followed from angiography until they experienced one of the primary endpoints; AMI (fatal or non-fatal), death or till December $31^{\text {st }} 2006$.

Information on clinical events was collected from The Western Norway Cardiovascular Registry and from the Cause of Death Registry at Statistics Norway as previously described [11]. An event was classified as fatal if death occurred within 28 days after onset. AMI was classified according to the diagnostic criteria of the revised AMI 
definition published in 2000 [16], and fatal strokes were classified according to diagnostic criteria published in 2001 [17]. Procedure-related non-fatal AMI occurring within $24 \mathrm{~h}$ of coronary angiography, PCI or CABG were not included in the end-point. CV death included causes of death coded I00-I99 or R96, according to the International Statistical Evaluation of Disease, Tenth Revision system. An endpoints committee adjudicated all events.

\section{Statistical analysis}

Continuous variables are presented as means (standard deviation (SD)). Categorical variables are reported as counts (percentage). Non-normally distributed variables (diastolic blood pressure, serum creatinine, CRP, plasma glucose, serum triglycerides and $\mathrm{Lp}(\mathrm{a})$ ) were $\log$ transformed. BMI groups were created using established BMI cut-offs; Normal weight (BMI 18.5-24.9 $\mathrm{kg} / \mathrm{m}^{2}$ ), overweight (BMI $\left.25-29.9 \mathrm{~kg} / \mathrm{m}^{2}\right)$ and obesity $\left(\mathrm{BMI} \geq 30 \mathrm{~kg} / \mathrm{m}^{2}\right)$. Underweight patients $(n=30)$ were eliminated due to the possibility of reverse causation. Between group differences were tested by one-way analysis of variance (ANOVA) or independent samples t-test for continuous variables, and by chi square test for categorical variables. Post hoc tests were applied for multiple comparisons where appropriate.

The relationships between baseline BMI and subsequent risk of AMI, CV death and all-cause mortality were evaluated across BMI groups. Hazard ratios (HR) and 95\% confidence intervals (CI) of endpoints associated with BMI categories were estimated with Cox proportional hazard models using the BMI normal weight category as reference. The time, in days, from angiography until endpoint (AMI, CV death and all-cause mortality) or end of study (December $31^{\text {st }}$ 2006) was used as time scale. Proportionality assumptions were tested by visual examination of $\log$ minus $\log$ plots and calculating Schoenfeld residuals. Covariates in the multivariate adjusted models were selected based on clinical relevance and the changein-estimate method [18], with a limit for inclusion of $10 \%$ change in the risk ratio. The final multivariate model included gender, age (continuous), LVEF (\%), current smoking (yes/no), angiotensin converting enzyme (ACE) -inhibitors (yes/no), loop diuretics (yes/no) and pulmonary disease (yes/no). Further adjustment of the multivariate Cox model did not alter the results in the total population or in gender stratified analyses; systolic and diastolic blood pressure $(\mathrm{mmHg})$, diabetes mellitus (yes/no), previous AMI (yes/no), extent of significant CAD $(0-3)$, serum creatinine levels $(\mu \mathrm{mol} / \mathrm{L}), \mathrm{CRP}(\mathrm{mg} / \mathrm{L})$, total cholesterol $(\mathrm{mmol} / \mathrm{L})$, vitamin B6 (yes/no) or folate/B12 (yes/no) intervention status (data not shown).

Effect modifications by gender were investigated by including the product of gender and BMI categories as an interaction term in the multivariate adjusted Cox model.
All tests were 2 -sided, and a p-value $<0.05$ was considered significant. Statistical analyses were performed with SPSS 17 (SPSS Inc, Chicago, IL) and R 2.14.2 (The RFoundation for Statistical Computing, Vienna, Austria).

\section{Results \\ Baseline characteristics}

The cohort consisted of 4131 patients (72\% males), and the mean (SD) age in the total population was 62 (10) years. Baseline characteristics across BMI groups are presented in Table 1. The mean (SD) BMI was 26.8 (3.9) $\mathrm{kg} / \mathrm{m}^{2}$, and $34 \%$ of the patients had a BMI within the normal weight range, 48\% were overweight and 19\% were obese.

Compared to the overweight and obese groups, the normal weight group was characterised by older age and a higher proportion of current smokers and subjects with a history of peripheral arterial disease. Mean blood pressure was lower in this group, as was the prevalence of diabetes. The extent of CAD at baseline did, however, not differ between the BMI categories. Compared to normal weight patients, overweight and obese patients were more often discharged with aspirin, statins and beta-blockers, while ACE inhibitors and loop diuretics were more often prescribed to obese patients.

The levels of eGFR, serum CRP, plasma glucose, HbA1c, $\mathrm{Hb}$, serum $\mathrm{ApoB}$, triglyceride and $\mathrm{Lp}(\mathrm{a})$ increased across incremental BMI groups, while serum ApoA1 and HDL cholesterol levels declined.

\section{Baseline characteristics according to gender}

Among men, 32\% were normal weight, 51\% overweight and $17 \%$ obese, whereas the respective proportions were $38 \%, 40 \%$ and $22 \%$ among women. Mean (SD) BMI was $26.8(3.7) \mathrm{kg} / \mathrm{m}^{2}$ among men and $26.8(4.7) \mathrm{kg} / \mathrm{m}^{2}$ among women.

Baseline characteristics according to gender and BMI groups are presented in Table 2. Compared to women, men were generally younger, and there was an inverse relationship between age and BMI among men. Men had worse $\mathrm{CV}$ risk profile and more severe CAD, at baseline, than women. Correspondingly, men, compared to women, did more often undergo revascularisation procedures following baseline angiography and were more often discharged with medication.

\section{Follow-up and end-points}

During the follow-up period (mean (SD) 4.8 (1.4) years), 337 (8.2\%) patients experienced an AMI, of which 101 (30\%) were fatal. A total of $302(7.3 \%)$ patients died, of whom 165 (55\%) died from cardiovascular causes.

There were statistically significant multivariate adjusted interactions between gender and BMI categories with regards to risk of incident AMI (p-int $=0.011)$ and 
Table 1 Baseline characteristics and laboratory findings according to BMI groups ${ }^{a}$

\begin{tabular}{|c|c|c|c|c|c|}
\hline & Total $n=4131$ & Normal weight $n=1395$ & Overweight $n=1970$ & Obese $n=766$ & p-value \\
\hline \multicolumn{6}{|l|}{ Demographic characteristics } \\
\hline Male sex, n (\%) & $2989(72.4)$ & $959(68.7)$ & $1517(77.0)$ & $513(67.0)$ & $<0.001$ \\
\hline Age (years) ${ }^{c}$ & $62(10)$ & $63(11)$ & $61(10)$ & $60(10)$ & $<0.001$ \\
\hline \multicolumn{6}{|l|}{ Clinical parameters } \\
\hline Systolic blood pressure (mmHg) & $141(20.7)$ & $139(21.0)$ & $142(20.6)$ & $143(20.3)$ & $<0.001$ \\
\hline Diastolic blood pressure $(\mathrm{mmHg})$ & $81.3(10.4)$ & $79.3(10.3)$ & $82.0(10.1)$ & $83.3(10.7)$ & $<0.001$ \\
\hline Left ventricular ejection fraction (\%) & $64.0(11.3)$ & $63.7(11.9)$ & $64.4(10.7)$ & $63.6(11.8)$ & 0.08 \\
\hline \multicolumn{6}{|l|}{ Cardiovascular risk factors, $\mathrm{n}(\%)$} \\
\hline Diabetes $^{d}$ & $496(12.0)$ & $123(8.8)$ & $209(10.6)$ & $164(21.4)$ & $<0.001$ \\
\hline Current smoker ${ }^{\mathrm{e}}$ & $1061(25.7)$ & $417(29.9)$ & $469(23.9)$ & $175(22.9)$ & $<0.001$ \\
\hline Ex smoker ${ }^{f}$ & $1933(46.9)$ & $571(40.6)$ & 975 (49.6) & $387(50.7)$ & $<0.001$ \\
\hline Family history of coronary heart disease ${ }^{g}$ & $1253(31.1)$ & $417(30.8)$ & $573(29.7)$ & $263(35.1)$ & 0.02 \\
\hline \multicolumn{6}{|l|}{ Cardiovascular history, $\mathrm{n}(\%)$} \\
\hline Previous acute myocardial infarction & $1670(40.4)$ & $564(40.4)$ & $784(39.8)$ & $322(42.0)$ & 0.56 \\
\hline Previous cerebrovascular disease & $286(6.9)$ & $100(7.2)$ & $128(6.5)$ & $58(7.6)$ & 0.55 \\
\hline Previous peripheral vascular arterial disease & $371(9.0)$ & $153(11.0)$ & $148(7.5)$ & $70(9.1)$ & $<0.01$ \\
\hline Previous percutaneous coronary intervention & $794(19.2)$ & $250(17.9)$ & $396(20.1)$ & $148(19.3)$ & 0.29 \\
\hline Previous coronary artery bypass graft surgery & $477(11.5)$ & $150(10.8)$ & $238(12.1)$ & 89 (11.6) & 0.49 \\
\hline \multicolumn{6}{|c|}{ Extent of coronary artery disease at baseline as assessed by coronary angiography, $n$ (\%) } \\
\hline No significant coronary artery disease & $1030(24.9)$ & $367(26.3)$ & $467(23.7)$ & $196(25.6)$ & 0.21 \\
\hline 1-vessel disease & $958(23.2)$ & $306(21.9)$ & $482(24.5)$ & $170(22.2)$ & 0.18 \\
\hline 2-vessel disease & $923(22.3)$ & $308(22.1)$ & $440(22.3)$ & $175(22.8)$ & 0.92 \\
\hline 3-vesseldisease & $1220(29.5)$ & $414(29.7)$ & $581(29.5)$ & $225(29.4)$ & 0.99 \\
\hline \multicolumn{6}{|l|}{ Comorbidity at baseline, $\mathrm{n}(\%)$} \\
\hline Pulmonary disease & $367(8.9)$ & $139(10.0)$ & $140(7.1)$ & $88(11.5)$ & $<0.001$ \\
\hline Cancer & $4(0.1)$ & $2(0.1)$ & $1(0.1)$ & $1(0.1)$ & 0.66 \\
\hline \multicolumn{6}{|c|}{ Medication following baseline coronary angiography, $n$ (\%) } \\
\hline Acetylsalisylic acid & $3376(81.7)$ & $1114(79.9)$ & $1638(83.1)$ & $624(81.5)$ & 0.05 \\
\hline Statins & $3310(80.1)$ & $1064(76.3)$ & $1626(82.5)$ & $620(80.9)$ & $<0.001$ \\
\hline$\beta$-blockers & $2994(72.5)$ & $970(69.5)$ & $1456(73.9)$ & $568(74.2)$ & 0.01 \\
\hline ACE inhibitors & $858(20.8)$ & $243(17.4)$ & $403(20.5)$ & $212(27.7)$ & $<0.001$ \\
\hline Loop diuretics & $447(10.8)$ & $127(9.1)$ & $182(9.2)$ & $138(18.0)$ & $<0.001$ \\
\hline \multicolumn{6}{|c|}{ Coronary revascularization following baseline coronary angiography, $n(\%)$} \\
\hline Percutaneous coronary intervention & $1348(32.6)$ & $432(31.0)$ & $663(33.7)$ & $253(33.0)$ & 0.26 \\
\hline Coronary artery bypass graft surgery & $892(21.6)$ & $302(21.6)$ & $438(22.2)$ & $152(19.8)$ & 0.39 \\
\hline \multicolumn{6}{|l|}{ Biochemical markers } \\
\hline Creatinine $(\mu \mathrm{mol} / \mathrm{L})$ & $92.6(31.1)$ & $92.5(33.4)$ & $93.0(32.0)$ & $91.8(23.4)$ & 0.34 \\
\hline eGFR (mL/min) & $87.8(17.3)$ & $86.2(17.7)$ & $88.5(16.6)$ & $88.9(17.8)$ & $<0.001$ \\
\hline CRP (mg/L) & $3.69(7.17)$ & $3.34(7.93)$ & $3.59(6.67)$ & $4.60(6.85)$ & $<0.001$ \\
\hline Glucose (mmol/L) & $6.35(2.40)$ & $5.93(2.24)$ & $6.32(2.28)$ & $7.20(2.79)$ & $<0.001$ \\
\hline $\mathrm{HbA1c}(\mathrm{mmol} / \mathrm{L})$ & $6.22(1.38)$ & $6.12(1.28)$ & $6.18(1.41)$ & $6.51(1.45)$ & $<0.001$ \\
\hline Hemoglobin (g/dL) & $14.2(1.24)$ & $13.9(1.24)$ & $14.4(1.18)$ & $14.4(1.29)$ & $<0.001$ \\
\hline ApoA1 (g/L) & $1.32(0.27)$ & $1.37(0.29)$ & $1.30(0.25)$ & $1.27(0.26)$ & $<0.001$ \\
\hline ApoB (g/L) & $0.90(0.25)$ & $0.87(0.24)$ & $0.91(0.24)$ & $0.93(0.26)$ & $<0.001$ \\
\hline
\end{tabular}


Table 1 Baseline characteristics and laboratory findings according to BMI groups ${ }^{\text {a }}$ (Continued)

\begin{tabular}{|c|c|c|c|c|c|}
\hline Total cholesterol (mmol/L) & $5.07(1.17)$ & $5.02(1.14)$ & $5.08(1.18)$ & $5.10(1.19)$ & 0.13 \\
\hline LDL cholesterol (mmol/L) & $3.09(1.03)$ & $3.05(1.02)$ & $3.12(1.00)$ & $3.11(1.10)$ & 0.11 \\
\hline HDL cholesterol (mmol/L) & $1.29(0.38)$ & $1.42(0.43)$ & $1.25(0.34)$ & $1.17(0.32)$ & $<0.001$ \\
\hline Triglycerides (mmol/L) & $1.78(1.22)$ & $1.43(0.90)$ & $1.86(1.28)$ & $2.22(1.39)$ & $<0.001$ \\
\hline Lp(a) (mmol/L) & $0.42(0.39)$ & $0.41(0.39)$ & $0.43(0.39)$ & $0.42(0.39)$ & 0.03 \\
\hline WENBIT intervention trial, $\mathrm{n}(\%)$ & $2560(62.0)$ & $797(57.1)$ & $1283(65.1)$ & $480(62.7)$ & $<0.001$ \\
\hline B6, n (\% of WENBIT participants) & $1275(49.8)$ & $394(49.4)$ & $657(51.2)$ & $224(46.7)$ & $<0.01$ \\
\hline Folate/B12, n (\% of WENBIT participants) & $1282(50.1)$ & $409(51.3)$ & $649(50.6)$ & $224(46.7)$ & 0.04 \\
\hline
\end{tabular}

$\mathrm{ACE}$, angiotensin converting enzyme; ApoA1, apolipoprotein A1; ApoB, apolipoprotein B; BMI, body mass index; CRP, c-reactive protein; eGFR, esitimated glomerular filtration rate; $H D L$, high density lipoprotein; $L D L$, low density lipoprotein; $L p(a)$, lipoprotein (a).

${ }^{\mathrm{a}}$ Normal weight (BMI $18.5-24.9 \mathrm{~kg} / \mathrm{m}^{2}$ ), overweight (BMI $25-29.9 \mathrm{~kg} / \mathrm{m}^{2}$ ) and obese $\left(\mathrm{BMI} \geq 30 \mathrm{~kg} / \mathrm{m}^{2}\right)$.

${ }^{\mathrm{b}}$ Based on between group differences calculated by one-way analysis of variance (ANOVA) for continuous variables and Chi squared test for categorical variables.

${ }^{\mathrm{c}}$ Mean (SD).

IIncludes DM type 1 and 2.

e Smokers included self-reported current smoking, those who quit smoking within $<1$ month and patients with plasma cotinine $>85 \mathrm{ng} / \mathrm{mL}$.

${ }^{f}$ Patients reported to have quit smoking $>1$ month prior to inclusion.

Included those reporting to have at least one 1st degree relative suffering from CAD before the age of 55 for men and 65 for women.

CV-death (p-int $=0.031)$, but not to all-cause mortality $(\mathrm{p}$-int $=0.427)$.

A total of $115(8.2 \%)$ normal weight patients, 127 (6.4\%) overweight patients and $60(7.8 \%)$ obese patients died. The risk of all-cause mortality did not differ significantly between BMI categories in any analyses; compared to the normal weight group, the multivariate adjusted HR (95\% CI) was $0.95(0.74,1.23)$ in the overweight group and 1.16 $(0.84,1.60)$ in the obese group.

Analyses were repeated in subgroups of patients with significant $\mathrm{CAD}$ or without diabetes only, and the results were not significantly different from those reported (data not shown).

\section{Gender stratified analyses}

A total of $267(8.9 \%)$ men and $70(6.1 \%)$ women suffered an AMI. Further, 241 (8.1\%) men and 61 (5.3\%) women died, whereof 132 (55\%) male deaths and 33 (54\%) female deaths were characterised as $\mathrm{CV}$ deaths.

Obese men had a significantly higher multivariate adjusted risk of both incident AMI; HR $1.80(1.28,2.52)$, and CV death; HR 1.60 (1.00, 2.55), compared to normal weight men (Table 3 ).

Overweight women had a significantly lower multivariate adjusted risk of AMI; HR $0.56(0.33,0.98)$, compared to normal weight women (Table 4). By contrast, the multivariate adjusted HR for AMI between normal weight women and obese women did not differ significantly.

\section{Discussion}

\section{Principal findings}

In this large longitudinal prospective cohort study of more than 4000 patients with suspected stable angina pectoris, we demonstrate that obese male patients had a 1.8 fold and 1.6 fold increased risk of incident AMI and $\mathrm{CV}$ death compared to normal weight men. By contrast, compared to normal weight women, obese women had similar risk of AMI and CV death, while overweight women had nearly half the risk of incident AMI. The risk of all-cause mortality associated with BMI was similar among men and women, and did not differ significantly across BMI categories.

\section{$\mathrm{BMI}$ and risk of $\mathrm{AMI}, \mathrm{CV}$ death and all-cause mortality in men and women}

Strong associations between overweight/obesity and risk of CVD and death have been demonstrated in the general population $[19,20]$. By contrast, several studies of patients with CAD have demonstrated that overweight and/or obese patients may have a better morbidity and mortality prognosis than their leaner counterparts; although as one recent review points out, this observation is not supported by all [3].

Only a few studies of patients with CAD have examined the association between BMI and risk of $\mathrm{CV}$ events and mortality in men and women separately. Our finding of an increased risk of cardiovascular events among obese men are in accordance with the results from a previous US study of patients with stable CVD, whereof $85 \%$ had CHD, as well as with a multi-ethnic sample study of patients with established CAD $[5,6]$. However, while these studies did not observe any significant associations between BMI and risk of major adverse coronary events in women, we report a nearly halved adjusted risk of AMI among overweight women as compared to their normal weight counterparts.

In the present study, there was no interaction between BMI and gender with regards to all-cause mortality. Moreover, the risk of death did not differ between BMI groups. These findings are in accordance with a previous study conducted among European patients with CAD [21]. To the best of our knowledge only two studies, 
Table 2 Baseline characteristics and laboratory findings according to gender and BMI groups ${ }^{\mathrm{a}}$

\begin{tabular}{|c|c|c|c|c|c|c|c|c|c|c|c|}
\hline & \multicolumn{4}{|c|}{ Men } & \multirow[b]{2}{*}{$p$-value ${ }^{b}$} & \multicolumn{4}{|c|}{ Women } & \multirow[b]{2}{*}{ p-value ${ }^{c}$} & \multirow[b]{2}{*}{$p$-value ${ }^{c}$} \\
\hline & $\begin{array}{c}\text { Total } \\
n=2989\end{array}$ & $\begin{array}{c}\text { Normal weight } \\
n=959\end{array}$ & $\begin{array}{c}\text { Overweight } \\
n=1517\end{array}$ & $\begin{array}{c}\text { Obese } \\
n=513\end{array}$ & & $\begin{array}{c}\text { Total } \\
n=1142\end{array}$ & $\begin{array}{c}\text { Normal weight } \\
n=436\end{array}$ & $\begin{array}{c}\text { Overweight } \\
n=453\end{array}$ & $\begin{array}{c}\text { Obese } \\
n=253\end{array}$ & & \\
\hline \multicolumn{12}{|l|}{ Demographic characteristics } \\
\hline Age $\left(\right.$ years) ${ }^{e}$ & $61(10)$ & $63(11)$ & $61(10)$ & $59(10)$ & $<0.001$ & $63(10)$ & $63(19)$ & $64(11)$ & $63(10)$ & 0.52 & $<0.001$ \\
\hline \multicolumn{12}{|l|}{ Clinical parameters } \\
\hline Systolic blood pressure (mmHg) & $141(20.4)$ & $140(20.5)$ & $141(20.3)$ & $143(20.4)$ & 0.03 & $141(21.6)$ & $138(22.1)$ & $143(21.6)$ & $144(20.2)$ & $<0.01$ & 0.94 \\
\hline Diastolic blood pressure $(\mathrm{mmHg})$ & $81.8(10.4)$ & $79.6(10.1)$ & $82.2(10.0)$ & $84.3(11.1)$ & $<0.001$ & $80.0(10.3)$ & $78.4(10.8)$ & $80.9(10.1)$ & $81.0(9.60)$ & $<0.001$ & $<0.001$ \\
\hline Left ventricular ejection fraction (\%) & $63.1(11.8)$ & $62.6(12.4)$ & $63.7(11.1)$ & $62.1(12.4)$ & $<0.01$ & $66.5(9.81)$ & $65.9(10.3)$ & $66.9(9.21)$ & $66.8(9.93)$ & 0.30 & $<0.001$ \\
\hline \multicolumn{12}{|l|}{ Cardiovascular risk factors, $\mathrm{n}(\%)$} \\
\hline Diabetes $^{f}$ & $361(12.1)$ & $97(10.1)$ & $158(10.4)$ & $106(20.7)$ & $<0.001$ & $135(11.8)$ & $26(6.0)$ & $51(11.3)$ & $58(22.9)$ & $<0.001$ & 0.82 \\
\hline Current smoker $^{9}$ & $803(26.9)$ & $305(31.8)$ & $375(24.8)$ & $123(24.0)$ & $<0.001$ & $258(22.7)$ & $112(25.7)$ & $94(20.8)$ & $52(22.7)$ & 0.16 & $<0.01$ \\
\hline Ex smoker ${ }^{h}$ & $1595(53.4)$ & $448(46.7)$ & $842(55.6)$ & $305(59.6)$ & $<0.001$ & $338(29.7)$ & $123(28.2)$ & $133(29.5)$ & $82(32.7)$ & 0.47 & $<0.001$ \\
\hline Family history of coronary heart disease $\mathrm{e}^{\mathrm{i}}$ & $844(29.0)$ & $258(27.8)$ & $417(28.1)$ & $169(33.9)$ & 0.03 & 409 (36.4) & $159(37.3)$ & $156(34.9)$ & $94(37.6)$ & 0.69 & $<0.001$ \\
\hline \multicolumn{12}{|l|}{ Cardiovascular history, n (\%) } \\
\hline Previous acute myocardial infarction & $1346(45.9)$ & $429(44.7)$ & $668(44.0)$ & $249(48.5)$ & 0.20 & $324(28.4)$ & $135(31.0)$ & $116(25.6)$ & $73(28.9)$ & 0.21 & $<0.001$ \\
\hline Previous cerebrovascular disease & $203(6.9)$ & $69(7.2)$ & $101(6.7)$ & $33(6.4)$ & 0.82 & $83(7.3)$ & $31(7.1)$ & $27(6.0)$ & $25(9.9)$ & 0.16 & 0.59 \\
\hline Previous peripheral vascular arterial disease & $276(9.2)$ & $114(11.9)$ & $115(7.6)$ & $47(9.2)$ & $<0.01$ & $95(8.3)$ & $39(8.9)$ & $33(7.3)$ & $23(9.1)$ & 0.59 & 0.36 \\
\hline Previous percutaneous coronary intervention & $641(21.4)$ & $193(20.1)$ & $340(22.4)$ & $108(21.1)$ & 0.39 & $153(13.4)$ & $57(13.1)$ & $56(12.4)$ & $40(15.8)$ & 0.42 & $<0.001$ \\
\hline Previous coronary artery bypass graft surgery & $394(13.2)$ & $116(12.1)$ & $205(13.5)$ & $73(14.2)$ & 0.44 & $83(7.3)$ & $34(7.8)$ & $33(7.3)$ & $16(6.3)$ & 0.77 & $<0.01$ \\
\hline \multicolumn{12}{|c|}{ Extent of coronary artery disease at baseline as assessed by coronary angiography, $n(\%)$} \\
\hline No significant coronary artery disease & $538(18.0)$ & $179(17.7)$ & $280(18.5)$ & $88(17.2)$ & 0.77 & $492(43.1)$ & $197(45.2)$ & $187(41.3)$ & $108(42.7)$ & 0.50 & $<0.001$ \\
\hline 1-vessel disease & $704(23.6)$ & $218(22.7)$ & $373(24.6)$ & $113(22.0)$ & 0.38 & $254(22.2)$ & $88(20.2)$ & $109(24.1)$ & $57(22.5)$ & 0.38 & 0.37 \\
\hline 2-vessel disease & $731(24.5)$ & $236(24.6)$ & $360(23.7)$ & $135(26.3)$ & 0.50 & $192(16.8)$ & $72(16.5)$ & $80(17.7)$ & $40(15.8)$ & 0.80 & $<0.001$ \\
\hline 3-vesseldisease & $1016(34.0)$ & $335(34.9)$ & $504(33.2)$ & $177(34.5)$ & 0.66 & $204(17.9)$ & $79(18.1)$ & $77(17.0)$ & $48(19.0)$ & 0.79 & $<0.001$ \\
\hline \multicolumn{12}{|l|}{ Comorbidity at baseline, $\mathrm{n}(\%)$} \\
\hline Pulmonary disease & $276(9.2)$ & $96(10.0)$ & $115(7.6)$ & $65(12.7)$ & 0.04 & $91(8.0)$ & $43(9.9)$ & $25(5.5)$ & $23(9.1)$ & $<0.01$ & 0.20 \\
\hline Cancer & $4(0.1)$ & $2(0.20)$ & $1(0.10)$ & $1(0.20)$ & 0.59 & $0(0.0)$ & 0 & 0 & 0 & - & 0.22 \\
\hline \multicolumn{12}{|c|}{ Medication following baseline coronary angiography, $\mathrm{n}(\%)$} \\
\hline Acetylsalisylic acid & $2546(85.2)$ & $810(84.5)$ & $1306(86.1)$ & $430(83.8)$ & 0.13 & $830(72.7)$ & $304(69.7)$ & $332(73.3)$ & $194(76.7)$ & 0.34 & $<0.001$ \\
\hline Statins & $2492(83.4)$ & $759(79.1)$ & $1296(85.4)$ & $437(85.2)$ & $<0.001$ & $818(71.6)$ & $305(70.0)$ & $330(72.8)$ & $183(72.3)$ & 0.61 & $<0.001$ \\
\hline$\beta$-blockers & $2229(74.6)$ & $684(71.3)$ & $1155(76.1)$ & $390(76.0)$ & 0.02 & $765(67.0)$ & $286(65.6)$ & $301(66.4)$ & $178(70.4)$ & 0.42 & $<0.001$ \\
\hline ACE inhibitors & $644(21.5)$ & $171(17.8)$ & $320(21.1)$ & $153(29.8)$ & $<0.001$ & $214(18.7)$ & $72(16.2)$ & $83(18.3)$ & $59(23.3)$ & 0.08 & 0.05 \\
\hline Loop diuretics & $297(9.9)$ & $90(9.4)$ & $128(8.4)$ & 79 (15.4) & $<0.001$ & $150(13.1)$ & $37(8.5)$ & $54(11.9)$ & $59(23.3)$ & $<0.001$ & $<0.01$ \\
\hline
\end{tabular}


Table 2 Baseline characteristics and laboratory findings according to gender and BMI groups ${ }^{\mathrm{a}}$ (Continued)

\begin{tabular}{|c|c|c|c|c|c|c|c|c|c|c|c|}
\hline \multicolumn{12}{|c|}{ Coronary revascularization following baseline coronary angiography, $\mathrm{n}(\%)$} \\
\hline Percutaneous coronary intervention & $1065(35.6)$ & $327(34.1)$ & $545(35.9)$ & $193(37.6)$ & 0.38 & $283(24.8)$ & $105(24.1)$ & $118(26.0)$ & $60(23.7)$ & 0.72 & $<0.001$ \\
\hline Coronary artery bypass graft surgery & $731(24.5)$ & $248(25.9)$ & $363(23.9)$ & $120(23.4)$ & 0.46 & $161(14.1)$ & $54(12.4)$ & $75(16.6)$ & $32(12.6)$ & 0.15 & $<0.001$ \\
\hline \multicolumn{12}{|l|}{ Biochemical markers } \\
\hline Creatinine $(\mu \mathrm{mol} / \mathrm{L})$ & $96.4(31.7)$ & $96.6(30.5)$ & $96.4(34.9)$ & $95.9(23.3)$ & 0.93 & $82.8(26.5)$ & $83.9(36.7)$ & $81.4(14.5)$ & 83.4 (21.5) & 0.35 & $<0.001$ \\
\hline eGFR (mL/min) & $89.3(17.0)$ & 87.5 (16.6) & 89.7 (16.6) & $91.2(16.9)$ & $<0.001$ & $84.1(17.3)$ & $83.4(17.6)$ & $84.6(16.1)$ & $84.2(18.7)$ & 0.58 & $<0.001$ \\
\hline $\mathrm{CRP}(\mathrm{mg} / \mathrm{L})$ & $3.66(7.59)$ & 3.55 (8.91) & 3.58 (6.99) & 4.07 (6.49) & 0.39 & $3.79(5.93)$ & $2.88(5.17$ & $3.62(5.43)$ & $5.66(7.42)$ & $<0.001$ & 0.59 \\
\hline Glucose (mmol/L) & $6.41(2.38)$ & $6.08(2.42)$ & $6.33(2.14)$ & $7.26(2.77)$ & $<0.001$ & $6.20(2.46)$ & $5.60(1.75)$ & $6.30(2.68)$ & $7.06(2.82)$ & $<0.001$ & 0.01 \\
\hline $\mathrm{HbA1c}(\mathrm{mmol} / \mathrm{L})$ & $6.17(1.39)$ & $6.16(1.11)$ & $6.40(1.52)$ & $6.62(1.49)$ & $<0.001$ & $6.36(1.38)$ & $6.10(1.36)$ & $6.11(1.37)$ & $6.46(1.43)$ & $<0.001$ & $<0.001$ \\
\hline Hemoglobin ( $\mathrm{g} / \mathrm{dL}$ ) & $14.6(1.16)$ & $14.2(1.21)$ & $14.7(1.07)$ & $14.8(1.16)$ & $<0.001$ & $13.4(1.05)$ & $13.3(1.04)$ & $13.5(1.04)$ & $13.5(1.05)$ & $<0.01$ & $<0.001$ \\
\hline ApoA1 (g/L) & $1.26(0.25)$ & $1.31(0.27)$ & $1.25(0.23)$ & $1.20(0.23)$ & $<0.001$ & $1.46(0.27)$ & $1.49(0.28)$ & $1.45(0.26$ & $1.42(0.27)$ & $<0.01$ & $<0.001$ \\
\hline ApoB (g/L) & $0.90(0.25)$ & $0.86(0.24)$ & $0.90(0.24)$ & $0.93(0.27)$ & $<0.001$ & $0.92(0.25)$ & $0.88(0.24)$ & $0.94(0.26)$ & $0.94(0.25)$ & $<0.01$ & $<0.01$ \\
\hline Total cholesterol (mmol/L) & $4.98(1.17)$ & $5.18(1.12)$ & $5.39(1.14)$ & $5.25(1.16)$ & 0.30 & $5.28(1.14)$ & $5.18(1.12)$ & $5.39(1.14)$ & $5.25(1.16)$ & 0.02 & $<0.001$ \\
\hline LDL cholesterol (mmol/L) & $3.05(1.02)$ & $3.03(1.01)$ & $3.06(0.98)$ & $3.07(1.13)$ & 0.64 & $3.19(1.03)$ & $3.08(1.02)$ & $3.30(1.03)$ & $3.18(1.04)$ & $<0.01$ & $<0.001$ \\
\hline $\mathrm{HDL}$ cholesterol (mmol/L) & $1.22(0.33)$ & $1.33(0.38)$ & $1.19(0.29)$ & $1.09(0.26)$ & $<0.001$ & $1.47(0.42)$ & $1.57(0.44)$ & $1.45(0.39)$ & $1.34(0.37)$ & $<0.001$ & $<0.001$ \\
\hline Triglycerides (mmol/L) & $1.85(1.28)$ & $1.48(0.97)$ & $1.91(1.30)$ & $2.32(1.52)$ & $<0.001$ & $1.62(1.03)$ & $1.34(0.72)$ & $1.67(1.18)$ & $2.00(1.07)$ & $<0.001$ & $<0.001$ \\
\hline $\mathrm{Lp}(\mathrm{a})(\mathrm{mmol} / \mathrm{L})$ & $0.41(0.37)$ & $0.43(0.41)$ & $0.48(0.45)$ & $0.47(0.40)$ & 0.79 & $0.46(0.42)$ & $0.43(0.41)$ & $0.48(0.45)$ & $0.47(0.40)$ & 0.11 & $<0.01$ \\
\hline WENBIT intervention trial, $\mathrm{n}(\%)$ & $2047(68.5)$ & $614(64.0)$ & $1072(70.7)$ & $361(70.4)$ & $<0.01$ & $513(44.9)$ & $183(42.0)$ & $211(46.6)$ & $119(47.0)$ & 0.29 & $<0.001$ \\
\hline B6, n (\% of WENBIT participants) & $1043(34.9)$ & $320(52.1)$ & $553(51.9)$ & $170(47.1)$ & 0.19 & $239(20.9)$ & 89 (48.6) & $96(45.5)$ & $54(45.4)$ & 0.94 & $<0.001$ \\
\hline Folate/B12, n (\% of WENBIT participants) & $1031(34.5)$ & $301(49.0)$ & $555(51.8)$ & $175(48.5)$ & 0.03 & $244(21.4)$ & $93(50.8)$ & $102(48.3)$ & $49(41.2)$ & 0.92 & $<0.001$ \\
\hline
\end{tabular}

$\overline{A C E}$, angiotensin converting enzyme; $A p o A 1$, apolipoprotein A1; ApoB, apolipoprotein B; BMI, body mass index; $C R P$, c-reactive protein; eGFR, esitimated glomerular filtration rate; $H D L$, high density lipoprotein; $L D L$, low density lipoprotein; $\mathrm{Lp}(\mathrm{a})$, lipoprotein (a).

${ }^{a}$ Normal weight (BMI $\left.18.5-24.9 \mathrm{~kg} / \mathrm{m}^{2}\right)$, overweight (BMI $\left.25-29.9 \mathrm{~kg} / \mathrm{m}^{2}\right)$ and obese $\left(B M I \geq 30 \mathrm{~kg} / \mathrm{m}^{2}\right)$.

bBased on differences between BMI groups among men, and was calculated by ANOVA for continuous variables and Chi squared test for categorical variables for differences between men and women.

'Based on differences between BMI groups among women, and was calculated by ANOVA for continuous variables and Chi squared test for categorical variables for differences between men and women.

${ }^{\mathrm{d}}$ Based on between group differences in men vs. women, and was calculated by independent samples t-test for continuous variables and Chi squared test for categorical variables.

${ }^{\mathrm{e}}$ Mean (SD).

Includes DM type 1 and 2.

${ }^{9}$ Smokers included self-reported current smoking, those who quit smoking within $<1$ month and patients with plasma cotinine $>85 \mathrm{ng} / \mathrm{mL}$.

hPatients reported to have quit smoking $>1$ month prior to inclusion.

'Included those reporting to have at least one 1st degree relative suffering from CAD before the age of 55 for men and 65 for women. 
Table 3 BMI groups ${ }^{a}$ and risk of acute myocardial infarction and cardiovascular death in men

\begin{tabular}{|c|c|c|c|c|c|c|c|c|}
\hline \multirow[t]{2}{*}{ Model } & \multicolumn{4}{|c|}{ Acute myocardial infarction } & \multicolumn{4}{|c|}{ Cardiovascular death } \\
\hline & Events, n (\%) & HR & $95 \% \mathrm{Cl}$ & p-value & Events, n (\%) & HR & $95 \% \mathrm{Cl}$ & p-value \\
\hline \multicolumn{9}{|l|}{ Univariate } \\
\hline Normal weight & $84(8.8)$ & 1.00 & & & $51(5.3)$ & 1.00 & & \\
\hline Overweight & $117(7.7)$ & 0.88 & $0.66,1.16$ & 0.37 & $49(3.2)$ & 0.61 & $0.41,0.90$ & 0.01 \\
\hline Obese & $66(12.9)$ & 1.51 & $1.09,2.08$ & 0.01 & $32(6.2)$ & 1.19 & $0.76,1.85$ & 0.45 \\
\hline \multicolumn{9}{|c|}{ Multivariate adjusted $^{\mathbf{b}}$} \\
\hline Normal weight & & 1.00 & & & & 1.00 & & \\
\hline Overweight & & 1.11 & $0.84,1.48$ & 0.47 & & 0.85 & $0.57,1.28$ & 0.44 \\
\hline Obese & & 1.80 & $1.28,2.52$ & $<0.01$ & & 1.60 & $1.00,2.55$ & 0.05 \\
\hline
\end{tabular}

$\mathrm{BMI}$, body mass index; $\mathrm{Cl}$, confidence interval; $\mathrm{HR}$, hazard ratio.

${ }^{a}$ Normal weight (BMI 18.5-24.9 kg/m ${ }^{2}$ ), overweight (BMI $25-29.9 \mathrm{~kg} / \mathrm{m}^{2}$ ) and obese $\left(\mathrm{BMI} \geq 30 \mathrm{~kg} / \mathrm{m}^{2}\right)$.

${ }^{\mathrm{b}} \mathrm{Age}$ (continuous), current smoking (yes/no), left ventricular ejection fraction (\%), pulmonary disease (yes/no), angiotensin converting enzyme-inhibitors (yes/no) and loop diuretics (yes/no).

of patients with CAD, have examined the association between BMI and all-cause mortality in men and women separately $[7,8]$. First, a study of Danish patients with AMI showed that normal weight, overweight and obese men had similar risk of death, whereas overweight women had a slightly decreased risk (HR (95\% CI); $0.78(0.68,0.90))$ of death, as compared to their normal weight counterparts. Furthermore, in a follow up study of the CADILLAC trial, they observed significantly lower in-hospital mortality ( $0.9 \%$ vs. $2.7 \%), 30$ days $(1.1 \%$ vs. $3.8 \%)$ and 1 - year (1.8\% vs. $7.5 \%)$ mortality in obese patients with AMI undergoing PCI when compared to normal weight patients. Statistical significance was, however, only reached in males.

\section{Possible explanations}

BMI is often used to quantify overweight and obesity owing to a high fat percentage correlation, but does not account for fat distribution. Men have a tendency to store excessive fat in visceral fat deposits, whereas women usually store fat in peripheral subcutaneous distributions [4]. Excessive visceral fat is associated with an increased risk of developing metabolic syndrome, putting men at a greater risk of developing CVD, while subcutaneous fat in the femoral-gluteal region may be associated with a more favourable CV risk profile [22]. Furthermore, overweight and obese postmenopausal women may benefit from the increase in circulating levels of estrogen produced by the adipose tissue [23,24].

Moreover, at baseline, men were more often affected by $\mathrm{CV}$ risk factors and had more severe CAD. Inclusion of these potential confounding variables in stratified multivariate analyses did not alter our results. Differing health status at baseline is thus unlikely to be the cause of the observed gender interaction.

\section{Strengths and limitations of the study}

The main strength of the present study is its, well defined population with complete follow up of clinical endpoints.

Table 4 BMI groups ${ }^{\mathrm{a}}$ and risk of acute myocardial infarction and cardiovascular death in women

\begin{tabular}{|c|c|c|c|c|c|c|c|c|}
\hline \multirow[t]{2}{*}{ Model } & \multicolumn{4}{|c|}{ Acute myocardial infarction } & \multicolumn{4}{|c|}{ Cardiovascular death } \\
\hline & Events, n (\%) & HR & $95 \% \mathrm{Cl}$ & p-value & Events, n (\%) & HR & $95 \% \mathrm{Cl}$ & p-value \\
\hline \multicolumn{9}{|l|}{ Univariate } \\
\hline Normal weight & $33(7.6)$ & 1.00 & & & $16(3.7)$ & 1.00 & & \\
\hline Overweight & $21(4.6)$ & 0.61 & $0.35,1.05$ & 0.08 & $13(2.9)$ & 0.78 & $0.38,1.63$ & 0.51 \\
\hline Obese & $16(6.3)$ & 0.84 & $0.46,1.53$ & 0.57 & $4(1.6)$ & 0.43 & $0.14,1.28$ & 0.13 \\
\hline \multicolumn{9}{|c|}{ Multivariate adjusted $^{b}$} \\
\hline Normal weight & & 1.00 & & & & 1.00 & & \\
\hline Overweight & & 0.56 & $0.33,0.98$ & 0.04 & & 0.71 & $0.34,1.50$ & 0.37 \\
\hline Obese & & 0.80 & $0.43,1.47$ & 0.46 & & 0.38 & $0.21,1.16$ & 0.09 \\
\hline
\end{tabular}

$\mathrm{BMI}$, body mass index; $\mathrm{Cl}$, confidence interval; $\mathrm{HR}$, hazard ratio.

${ }^{2}$ Normal weight (BMI $\left.18.5-24.9 \mathrm{~kg} / \mathrm{m}^{2}\right)$, overweight $(B M I) 25-29.9 \mathrm{~kg} / \mathrm{m}^{2}$ ) and obese $\left(\mathrm{BMI} \geq 30 \mathrm{~kg} / \mathrm{m}^{2}\right)$.

${ }^{b}$ Age (continuous), current smoking (yes/no), left ventricular ejection fraction (\%), pulmonary disease (yes/no), angiotensin converting enzyme-inhibitors (yes/no) and loop diuretics (yes/no). 
Limitations include the single baseline measurements of BMI and other time dependent cofactors such as medication. We did not have sufficient data on possible confounders such as physical activity, socioeconomic status or cardio- respiratory fitness and intentional vs. unintentional weight loss, and thus we cannot exclude the possibility that residual confounding from unmeasured causal factors unevenly distributed between BMI groups may have influenced our results. Unfortunately, we did not have data on recent weight loss prior to inclusion, but as underweight patients (BMI $<18.5 \mathrm{~kg} / \mathrm{m}^{2}$ ) were excluded, and adjustment for possible confounders such as cancer, pulmonary disease, extent of significant CAD and LVEF did not significantly alter our results, reverse causation is unlikely. BMI was positively associated with common obesity related characteristics such as higher blood pressure, diabetes, an unfavourable lipid profile, higher eGFR and CRP. Adjustment for these variables did not have a significant effect on our results, but we would in any case not include these variables in a final multivariate adjusted survival model because of the possibility of over-adjustment bias. We did, however, adjust for use of ACE inhibitors and loop diuretics as a proxy of heart failure, and there is the possibility that these variables may have mediated some of the effect of BMI.

It has previously been suggested that BMI is an inadequate marker of overweight and obesity in patients with CAD [25], with waist circumference or waist to hip ratio suggested as better predictors of cardiovascular events, especially in women $[6,26]$. Studies supporting an obesity paradox have almost exclusively used BMI as an index of obesity [3]. We thus suspect that the diverging findings among such studies may be the result of BMI's inadequacy as a quantifier of true body fatness and fat distribution.

Given that there were relatively few females in the study population and the event rate was low, we thus had a low statistical power to by which to detect the possible effects of BMI on risk of events among women. Further, we cannot rule out that the relatively lower incidence rate of AMI among women is a result of detection bias; women, compared to men, are more likely to experience atypical symptoms of AMI and may consequently delay seeking medical care for symptoms or be misdiagnosed by healthcare providers [27]. Finally, the inclusion of predominantly white subjects limits the ability to generalise our findings to non-white populations.

\section{Conclusion}

Among 4131 men and women with suspected stable angina pectoris, obese men carried an $80 \%$ and $60 \%$ higher risk of $\mathrm{AMI}$ and $\mathrm{CV}$ death, respectively, compared to normal weight men, whereas being overweight, compared to normal weight, was associated with a $50 \%$ lower risk of AMI among women. These findings may potentially explain some of the result variation in studies reporting on the obesity paradox, with further investigation of the interaction between gender and BMI in terms of risk of $\mathrm{CV}$ events and mortality therefore warranted.

\section{Competing interests}

The authors declare that they have no competing interests.

\section{Authors' contributions}

ON conceived of the study and contributed to the study design; GFTS, EKRP, $\mathrm{HSH}$ and ON conducted research; HB, RS analyzed data or performed statistical analysis; $\mathrm{HB}, \mathrm{JKH}, \mathrm{GFTS}, \mathrm{JH}$ and $\mathrm{ON}$ wrote the paper; $\mathrm{HB}$ had primary responsibility for final content; $\mathrm{HB}, \mathrm{JKH}, \mathrm{JH}$ and ON interpreted data; HB, JKH, GFTS, EKRP, HSH, $\mathrm{JH}$ and $\mathrm{ON}$ critically revised the manuscript. All listed authors take responsibility for all aspects of the reliability and freedom from bias of the data presented and their discussed interpretation. All authors read and approved the final manuscript.

\section{Acknowledgements}

We thank all WENBIT coworkers at Haukeland and Stavanger University Hospitals, Norway, as well as the Endpoints Committee: Marta Ebbing (HUS), Leik Woie (SUS), Eva Ringdal Pedersen (UiB), Hall Schartum-Hansen (UiB), Per Lund Johansen (UiB) (Chair). We would also like to thank all those who participated in the study for their time and effort. Thanks are also due to Matthew McGee for proofreading the manuscript.

\section{Author details}

'Morbid Obesity Center, Vestfold Hospital Trust, Tønsberg, Norway. ${ }^{2}$ Institute of Clinical Medicine, University of Oslo, Oslo, Norway. ${ }^{3}$ Department of Clinical Science, University of Bergen, Bergen, Norway. ${ }^{4}$ Department of Heart Disease, Haukeland University Hospital, Bergen, Norway.

Received: 21 November 2013 Accepted: 16 May 2014

Published: 21 May 2014

\section{References}

1. The top 10 causes of death. http://www.who.int/mediacentre/factsheets/ fs310/en/.

2. Goff DC Jr, Lloyd-Jones DM, Bennett G, Coady S, D'Agostino RB Sr, Gibbons R, Greenland P, Lackland DT, Levy D, O'Donnell CJ, Robinson J, Schwartz JS, Shero ST, Smith SC Jr, Sorlie P, Stone NJ, Wilson PWF: 2013 ACC/AHA guideline on the assessment of cardiovascular risk: a report of the American College of Cardiology/American Heart Association Task Force on Practice Guidelines. Circulation 2013,

3. Chrysant SG, Chrysant GS: New insights into the true nature of the obesity paradox and the lower cardiovascular risk. J Am Soc Hypertens 2013, 7(1):85-94.

4. Lemieux S, Prud'homme D, Bouchard C, Tremblay A, Despres JP: Sex differences in the relation of visceral adipose tissue accumulation to total body fatness. Am J Clin Nutr 1993, 58(4):463-467.

5. Domanski MJ, Jablonski KA, Rice MM, Fowler SE, Braunwald E, Investigators P: Obesity and cardiovascular events in patients with established coronary disease. Eur Heart J 2006, 27(12):1416-1422.

6. Dagenais GR, Yi Q, Mann JF, Bosch J, Pogue J, Yusuf S: Prognostic impact of body weight and abdominal obesity in women and men with cardiovascular disease. Am Heart J 2005, 149(1):54-60.

7. Kragelund C, Hassager C, Hildebrandt P, Torp-Pedersen C, Kober L: Impact of obesity on long-term prognosis following acute myocardial infarction. Int J Cardiol 2005, 98(1):123-131.

8. Nikolsky E, Stone GW, Grines CL, Cox DA, Garcia E, Tcheng JE, Griffin JJ, Guagliumi G, Stuckey T, Turco M, Negoita M, Lansky AJ, Mehran R: Impact of body mass index on outcomes after primary angioplasty in acute myocardial infarction. Am Heart J 2006, 151(1):168-175.

9. Wessel TR, Arant CB, Olson MB, Johnson BD, Reis SE, Sharaf BL, Shaw LJ, Handberg E, Sopko G, Kelsey SF, Pepine CJ, Merz NB: Relationship of physical fitness vs body mass index with coronary artery disease and cardiovascular events in women. JAMA 2004, 292(10):1179-1187.

10. Svingen GF, Ueland PM, Pedersen EK, Schartum-Hansen H, Seifert R, Ebbing M, Loland KH, Tell GS, Nygard O: Plasma dimethylglycine and risk of incident acute myocardial infarction in patients with stable angina pectoris. Arterioscler Thromb Vasc Biol 2013. 
11. Ebbing M, Bleie O, Ueland PM, Nordrehaug JE, Nilsen DW, Vollset SE, Refsum H, Pedersen EK, Nygard O: Mortality and cardiovascular events in patients treated with homocysteine-lowering B vitamins after coronary angiography: a randomized controlled trial. JAMA 2008, 300(7):795-804.

12. Benowitz NL, Jacob P III, Ahijevych K, Jarvis MJ, Hall S, LeHouezec J, Hansson A, Henningfield J, Tsoh J, Hurt RD, Velicer W for the The SRNT Subcommittee on Biochemical Verification: Biochemical verification of tobacco use and cessation. Nicotine Tob Res 2002, 4(2):149-159.

13. Midttun O, Hustad S, Ueland PM: Quantitative profiling of biomarkers related to B-vitamin status, tryptophan metabolism and inflammation in human plasma by liquid chromatography/tandem mass spectrometry. Rapid Commun Mass Spectrom 2009, 23(9):1371-1379.

14. Friedewald WT, Levy RI, Fredrickson DS: Estimation of the concentration of low-density lipoprotein cholesterol in plasma, without use of the preparative ultracentrifuge. Clin Chem 1972, 18(6):499-502.

15. Levey AS, Stevens LA, Schmid CH, Zhang YL, Castro AF 3rd, Feldman HI, Kusek JW, Eggers P, Van Lente F, Greene T, Coresh J: A new equation to estimate glomerular filtration rate. Ann Intern Med 2009, 150(9):604-612.

16. Alpert JS, Antman E, Apple F, Beller G, Breithardt G, Armstrong PW, Bassand JP, Baye's de Luna A, Chaitman BR, Clemmensen P, Falk E, Fishbein MC, Galvani M, Garson A Jr, Grines C, Hamm C, Hoppe U, Jaffe A, Katus H, Kjekshus J, Klein W, Klootwijk P, Lenfant C, Levy D, Levy RI, Luepker R, Marcus F, Näslund U, Ohman $M$, Pahlm O, et al: Myocardial infarction redefined-a consensus document of The Joint European Society of Cardiology/American College of Cardiology Committee for the redefinition of myocardial infarction. Eur Heart J 2000, 21(18):1502-1513.

17. Cannon CP, Battler A, Brindis RG, Cox JL, Ellis SG, Every NR, Flaherty JT, Harrington RA, Krumholz HM, Simoons ML, Van De Werf FJJ, Weintraub WS, Mitchell KR, Morrisson SL, Anderson HV, Cannom DS, Chitwood WR, Cigarroa JE, Collins-Nakai RL, Gibbons RJ, Grover FL, Heidenreich PA Khandheria BK, Knoebel SB, Krumholz HL, Malenka DJ, Mark DB, Mckay CR, Passamani ER, Radford MJ, et al: American College of Cardiology key data elements and definitions for measuring the clinical management and outcomes of patients with acute coronary syndromes. A report of the American College of Cardiology Task Force on Clinical Data Standards (Acute Coronary Syndromes Writing Committee). J Am Coll Cardiol 2001, 38(7):2114-2130.

18. Greenland S: Modeling and variable selection in epidemiologic analysis. Am J Public Health 1989, 79(3):340-349.

19. Grundy SM, Pasternak R, Greenland P, Smith S Jr, Fuster V: Assessment of cardiovascular risk by use of multiple-risk-factor assessment equations: a statement for healthcare professionals from the American Heart Association and the American College of Cardiology. Circulation 1999, 100(13):1481-1492.

20. Eckel RH: Obesity and heart disease: a statement for healthcare professionals from the Nutrition Committee. Am Heart Assoc Circul 1997, 96(9):3248-3250

21. De Bacquer D, De Backer G, Ostor E, Simon J, Pyorala K, Group EIS: Predictive value of classical risk factors and their control in coronary patients: a follow-up of the EUROASPIRE I cohort. Eur J Cardiovasc Prev Rehabil 2003, 10(4):289-295.

22. Snijder MB, van Dam RM, Visser M, Seidell JC: What aspects of body fat are particularly hazardous and how do we measure them? Int J Epidemiol 2006, 35(1):83-92

23. Silva TC, Barrett-Connor E, Ramires JA, Mansur AP: Obesity, estrone, and coronary artery disease in postmenopausal women. Maturitas 2008, 59(3):242-248

24. Castracane VD, Kraemer GR, Ogden BW, Kraemer RR: Interrelationships of serum estradiol, estrone, and estrone sulfate, adiposity, biochemical bone markers, and leptin in post-menopausal women. Maturitas 2006, 53(2):217-225

25. Romero-Corral A, Somers VK, Sierra-Johnson J, Jensen MD, Thomas RJ, Squires RW, Allison TG, Korinek J, Lopez-Jimenez F: Diagnostic performance of body mass index to detect obesity in patients with coronary artery disease. Eur Heart J 2007, 28(17):2087-2093.

26. Rexrode KM, Carey VJ, Hennekens CH, Walters EE, Colditz GA, Stampfer MJ, Willett WC, Manson JE: Abdominal adiposity and coronary heart disease in women. JAMA 1998, 280(21):1843-1848.
27. Maas AH, van der Schouw YT, Regitz-Zagrosek V, Swahn E, Appelman YE, Pasterkamp G, Ten Cate H, Nilsson PM, Huisman MV, Stam HC, Eizema K, Stramba-Badiale M: Red alert for women's heart: the urgent need for more research and knowledge on cardiovascular disease in women: proceedings of the workshop held in Brussels on gender differences in cardiovascular disease, 29 September 2010. Eur Heart J 2011, 32(11):1362-1368.

doi:10.1186/1471-2261-14-68

Cite this article as: Borgeraas et al:: Association of body mass index with risk of acute myocardial infarction and mortality in Norwegian male and female patients with suspected stable angina pectoris: a prospective cohort study. BMC Cardiovascular Disorders 2014 14:68.

\section{Submit your next manuscript to BioMed Central and take full advantage of:}

- Convenient online submission

- Thorough peer review

- No space constraints or color figure charges

- Immediate publication on acceptance

- Inclusion in PubMed, CAS, Scopus and Google Scholar

- Research which is freely available for redistribution

Submit your manuscript at www.biomedcentral.com/submit
C) BioMed Central 